\title{
GEOPHYSICS
}

\section{Micro-seismic event estimation and velocity analysis based on a source-focusing function}

\begin{tabular}{|r|l|}
\hline Journal: & Geophysics \\
\hline Manuscript ID & GEO-2018-0205.R3 \\
\hline Manuscript Type: & Technical Paper \\
\hline Keywords: & imaging, inversion, microseismic, sources, passive seismic \\
\hline Area of Expertise: & Passive Seismic and Microseismic Methods, Seismic Inversion \\
\hline \multicolumn{2}{|l}{} \\
\end{tabular}

\section{SCHOLARONE ${ }^{\mathrm{m}}$ Manuscripts}




\title{
Micro-seismic event estimation and velocity analysis based
} on a source-focusing function

\author{
Chao Song, Tariq Alkhalifah, Zedong Wu \\ King Abdullah University of Science and Technology (KAUST), \\ Physical Sciences and Engineering Division, \\ Thuwal, Saudi Arabia, 23955 \\ (January 2, 2019) \\ Running head: Micro-seismic event estimation
}

\begin{abstract}
Attaining information corresponding to the micro-seismic source location helps in understanding the reservoir fracturing process. Time-reversal-based migration methods are widely used to obtain a source image directly. Such source-locating methods share a fundamental weakness: the accuracy of source image depends highly on the accuracy of the velocity model. Full waveform inversion (FWI) has been employed in micro-seismic data to include an optimization of the source image and velocity model, simultaneously. However, such inversions are vulnerable to cycle-skipping problems, more so when the source location and source origin time are unknown. The computational cost of FWI is also high. In order to solve these problems, we introduce a source-focusing function as an additional objective to optimize the velocity model and source image. This objective function used to measure the source-image focusing property is defined by the estimated source-location coordinate and the source image. The source image is poorly focused if the velocity is inaccurate, and the objective function reaches a minimum when the velocity is accurate. We use the geometric-mean imaging condition to get a high-resolution source image at each iteration.
\end{abstract}


Then, we use this source image to calculate the estimated source-location coordinate and the gradient of the objective functional with respect to the velocity. The optimized velocity can improve the source-image quality. In the end, the final output velocity and source image will allow us to fit the objective for all these attributes of the model and source image. Using synthetic data generated from the 2D Marmousi and the 3D Overthrust models, we highlight the cycle-skipping immune and high-resolution features of the proposed method. The application to field data also shows that the proposed method can improve the velocity model and source-image quality. 


\section{INTRODUCTION}

Hydraulic fracturing is an important procedure in oil and gas extraction. In order to make dense rock crack, liquids or $\mathrm{CO}_{2}$ are injected into the reservoir area to create high-pressure conditions. The micro-seismic events caused by the cracking of the rock can be monitored through sensors in the well or on the Earth's surface. Locating micro-seismic events can help us understand the fracking impact, as we map the hydraulic fracturing, and monitor potential reservoir fluid migrations and production (Warpinski, 2009; Lin and Zhang, 2016). Such information will help the engineers make better injection decisions and strategies.

Over the years, researchers have developed many methods to locate micro-seismic sources. Ray-based methods rely on the traveltime picking accuracy of either P-wave or S-wave arrivals (Waldhauser and Ellsworth, 2000; Eisner et al., 2009). Though efficient and reasonably reliable, this category of methods tend to fail when the signal-to-noise ratio (SNR) is low, which results in inaccurate traveltime picking. These methods also fail areas with complex velocity structure. In order to mitigate the uncertainty from the event picking, time-reversal (Gajewski and Tessmer, 2005; Artman et al., 2010; Sava, 2011) and Gaussian-beam migration (Rentsch et al., 2004) based methods were developed. These methods adhere to the concept of wavefield reconstruction backward in time. By using an appropriate imaging condition, micro-seismic source images are obtained. This category of methods requires denser acquisition scenarios compared to traveltime methods. Specially designed source-imaging conditions, like the interferometric imaging condition and the geometric-mean imaging condition were proposed to achieve micro-seismic monitoring with sparsely sampled and noisy data (Sava, 2011; Nakata and Beroza, 2016). By matching the observed data with a demigrated version of the data using non-stationary filters, Song et al. (2017) managed to 
develop filters that improve the resolution of the source image. However, these kinds of methods also have several limitations. The uncertainties of the source wavelet and source origin time make the wavefield-reconstruction result unreliable, and such information is usually unavailable in micro-seismic case. Velocity accuracy is another key factor to guarantee the success of constructing the source image. In order to solve these problems, full waveform inversion (FWI) has recently been utilized to estimate micro-seismic events. Kaderli et al. (2015) proposed to separate the wave equation source term into spatial and temporal components. They used FWI to invert for the source components. Wang and Alkhalifah (2016) developed a source-independent FWI method to invert for the velocity model, as well as the micro-seismic source image. By convolving reference traces with the observed and modeled data (Choi and Alkhalifah, 2011), the effect of the unknown source origin time can be mitigated. By inverting the source wavelet and the velocity model, source location can be accurately determined. Wu and Alkhalifah (2017) proposed a source-extension approach to determine the source location while inverting for the velocity and source image simultaneously in the frequency domain. Some researchers also developed feasible strategies to incorporate time-reversal source imaging and FWI to get good velocity models and source images (Sun et al., 2016). Compared with time-reversal-based methods, FWI can truly provide more information like the velocity and source components. However, the computational cost and cycle-skipping problems associated with FWI have limited its success in practical applications. Witten and Shragge (2017) proposed to separate P- and S-wave modes and calculate the source image by autocorrelating the adjoint P- and S-wavefields. This method requires extensions in both the time and space coordinates, so the computational cost is also high.

In this paper, we present a new method to optimize the velocity and the source image 
iteratively. The velocity accuracy is directly related to the focusing property of the source image. Taking advantage of this physical feature, we propose to use a source-energy focusing measurement function as the objective function instead of matching the observed and calculated data directly like in the conventional FWI (Wu and Alkhalifah, 2017). This objective function consists of two variables: source-location coordinate and the source image. It can also be used as a penalty function to enforce the focusing property of the source image which is discussed in Song et al. (2019). With the help of a new objective function, we no longer need to worry about the cycle-skipping problem and a bad initial source-location guess in FWI. What is more, since we no longer need to invert for the source image over many iterations, this method is reasonably efficient. We use the geometric-mean condition to produce the source image at each iteration (Nakata and Beroza, 2016). This source imaging condition yields high-resolution source images even with sparsely collected data. This means that this imaging condition can make the source image more focused regardless of the limits of the acquisition system. In addition, this source-imaging condition can also effectively suppress the noise in the data. As we update the velocity through iterations with a conjugate gradient method, the source-image quality is also improved gradually as part of the objective. Finally, we test the proposed method on synthetic data generated from the 2D Marmousi model, and a 3D Overthrust model. We also test the proposed method on field data.

\section{THEORY}

Wave propagation in isotropic acoustic media with constant density from a point source can be modeled by solving the following wave equation:

$$
\frac{1}{v^{2}} \frac{\partial^{2} u}{\partial t^{2}}-\nabla^{2} u=f(t) \delta\left(\mathbf{x}-\mathbf{x}_{\mathbf{s}}\right)
$$


where $v$ is the velocity, $f(t)$ is the wavelet function, $\mathbf{x}=[x, y, z]$ denotes spatial dimensions, $\mathbf{x}_{\mathbf{S}}$ is the source coordinate, and $u(\mathbf{x}, t)$ is the wavefield. For FWI to be successful using a point-source wave equation, Huang and Symes (2016) proposed to use an extended source function to replace the point source. By doing this, nonphysical source energy away from $\mathbf{x}=\mathbf{x}_{\mathbf{s}}$ can be penalized. This idea can also be used to quantitatively measure the source energy concentration property of the source image. By taking advantage of this property, the objective function for optimizing the source image is given as:

$$
E\left(v, \mathbf{x}_{\mathbf{s}}\right)=\sum_{\mathbf{x}} \frac{1}{2}\left\|\left|\mathbf{x}-\mathbf{x}_{\mathbf{s}}\right| I[v]\right\|_{2}^{2}
$$

$I[v]$ represents the source image calculated with the geometrical-mean imaging condition (Nakata and Beroza, 2016). In active seismic inversion, researchers developed the migration velocity-analysis (MVA) method based on differential semblance optimization (DSO) in the subsurface-offset domain (Hou and Symes, 2018). The basic idea of this method is to penalize the energy outside the zero-offset panel to focus the offset gather. Similarly, our method aims to penalize the source energy away from $\mathbf{x}_{\mathbf{s}}$ to make the source image more focused. First, we divide the recorded data into different receiver groups (Sun et al., 2016). The way we set receiver groups is shown in Figure 1. Receivers with the same color belong to the same receiver group, and receivers from different groups are set adjacently in the fixed order. Then, we construct the adjoint wavefields from the different receiver groups, as:

$$
\frac{1}{v_{0}^{2}} \frac{\partial^{2} \lambda_{i}}{\partial t^{2}}=\nabla^{2} \lambda_{i}+d_{i}\left(\mathbf{x}_{\mathbf{r}}, T-t\right),
$$

where index $i$ represents the receiver-group number, $d_{i}\left(\mathbf{x}_{\mathbf{r}}, T-t\right)$ represents the recorded data of the $i$ th receiver group at receiver locations $\mathbf{x}_{\mathbf{r}}, \lambda_{i}(t, v)$ represents the adjoint wavefield 
calculated with background velocity $v_{0}$. Then we multiply all these wavefields and integrate over time to obtain the source image (Nakata and Beroza, 2016):

$$
I[v]=\int_{t} \prod_{i=1}^{n} \lambda_{i}(t, v) d t .
$$

In equation 4 , continued multiplication $\prod$ means multiplying all wavefields from different receiver groups, $n$ is the total number of receiver groups. We can easily notice that no source wavelet or source origin time are involved in this source-imaging condition. After getting the source image, we can calculate the source location by setting $\frac{\partial E}{\partial \mathbf{x}_{\mathbf{s}}}=0$. As a result, the analytical solution of $\mathbf{x}_{\mathbf{s}}$ is derived as:

$$
\mathbf{x}_{\mathbf{s}}=\frac{\sum_{\mathbf{x}} I[v]^{2} \mathbf{x}}{\sum_{\mathbf{x}} I[v]^{2}}
$$

According to the Born theory, the perturbed wavefield $\delta \lambda_{i}$ is:

$$
\frac{1}{v_{0}^{2}} \frac{\partial^{2} \delta \lambda_{i}}{\partial t^{2}}=\nabla^{2} \delta \lambda_{i}+\frac{2 \delta v}{v_{0}^{3}} \frac{\partial^{2} \lambda_{i}}{\partial t^{2}}
$$

Equation 6 can be written in an abbreviated form as: $L \delta \lambda_{i}=\frac{2 \delta v}{v_{0}^{3}} \frac{\partial^{2} \lambda_{i}}{\partial t^{2}}$, where $L=\frac{1}{v_{0}^{2}} \frac{\partial^{2}}{\partial t^{2}}-\nabla^{2}$ is the modeling/de-migration operator. Using the adjoint-state method, we can perturb the objective function with respect to a perturbation in velocity, $\delta v$, as follows (Plessix, 2006):

$$
\begin{aligned}
\delta E & =\sum_{\mathbf{x}}\left|\mathbf{x}-\mathbf{x}_{\mathbf{s}}\right|^{2} I[v] \delta I[v] \\
& =\sum_{j=1}^{n} \sum_{\mathbf{x}}\left|\mathbf{x}-\mathbf{x}_{\mathbf{s}}\right|^{2} I[v] \int_{t} \prod_{i=1, i \neq j}^{n} \lambda_{i}(t, v) \delta \lambda_{j}(t, v) d t \\
& =\sum_{j=1}^{n} \int_{t}\left\langle\sum_{\mathbf{x}}\left|\mathbf{x}-\mathbf{x}_{\mathbf{s}}\right|^{2} I[v] \int_{\zeta} \prod_{i=1, i \neq j}^{n} \lambda_{i}(\zeta, v) d \zeta, L^{-1}\left(\frac{2 \delta v}{v^{3}} \frac{\partial^{2} \lambda_{j}(t, v)}{\partial t^{2}}\right)\right\rangle d t \\
& =\sum_{j=1}^{n} \int_{t}\left\langle L^{-T}\left\{\sum_{\mathbf{x}}\left|\mathbf{x}-\mathbf{x}_{\mathbf{s}}\right|^{2} I[v] \int_{\zeta} \prod_{i=1, i \neq j}^{n} \lambda_{i}(\zeta, v) d \zeta\right\}, \frac{2 \delta v}{v^{3}} \frac{\partial^{2} \lambda_{j}(t, v)}{\partial t^{2}}\right\rangle d t \\
& =\left\langle\sum_{j=1}^{n} \int_{t} L^{-T}\left\{\sum_{\mathbf{x}}\left|\mathbf{x}-\mathbf{x}_{\mathbf{s}}\right|^{2} I[v] \int_{\zeta} \prod_{i=1, i \neq j}^{n} \lambda_{i}(\zeta, v) d \zeta\right\} \frac{2}{v^{3}} \frac{\partial^{2} \lambda_{j}(t, v)}{\partial t^{2}} d t, \delta v\right\rangle,
\end{aligned}
$$


Based on above derivation, the velocity gradient $\nabla_{v} E$ is:

$$
\nabla_{v} E=\frac{2}{v^{3}} \sum_{j=1}^{n} \int_{t} L^{-T}\left\{\sum_{\mathbf{x}}\left|\mathbf{x}-\mathbf{x}_{\mathbf{s}}\right|^{2} I[v] \int_{\zeta} \prod_{i=1, i \neq j}^{n} \lambda_{i}(\zeta, v) d \zeta\right\} \cdot \frac{\partial^{2} \lambda_{j}(t, v)}{\partial t^{2}} d t
$$

In equation $8, \frac{\partial^{2} \lambda_{j}(t, v)}{\partial t^{2}}$ is the second-order derivative of the adjoint wavefield with respect to time, $\sum_{\mathbf{x}}\left|\mathbf{x}-\mathbf{x}_{\mathbf{s}}\right|^{2} I[v] \int_{\zeta} \prod_{i=1, i \neq j}^{n} \lambda_{i}(\zeta, v) d \zeta$ will act like a new source for a forward wavefield. The velocity gradient is the multiplication of these two terms. With the help of the conjugate gradient method, we update the background velocity using

$$
v_{0}=v_{0}-\alpha \nabla_{v} E
$$

where $\alpha$ is the step length we choose. Now we have an optimized velocity model which will lead to a more focused source image. So we simply repeat the steps shown in the equation $3,4,5,8$ and 9 , until convergence is achieved, and we can get the final result of the updated velocity model and the optimized source image. We summarize the proposed method in algorithm 1 to make it comprehensible. In this whole updating procedure, no data comparison and update is involved, so the cycle-skipping problem we often suffer in FWI is mitigated. We only need to calculate the source image and source-location coordinate, and update the velocity.

\section{NUMERICAL EXAMPLES}

\section{Source imaging}

First, we test the performance of the geometric-imaging condition for source imaging using a Marmousi model of size $384 \times 126$ samples, as shown in Figure 2a. The horizontal and vertical grid intervals are both $25 \mathrm{~m}$. We use a Ricker wavelet with a peak frequency of 6 $\mathrm{Hz}$ as a source wavelet. The true source location is $(6000 \mathrm{~m}, 2000 \mathrm{~m})$. All the grid points 
Algorithm 1 Micro-seismic event estimation and velocity analysis algorithm Input: Observed data $d_{o}$; Initial velocity model; Number of receiver group $n$; Number of iterations Niter.

Output: Final source image; inverted velocity model.

for iter=1:Niter; do

Calculate the source image using geometric-mean imaging condition $I[v]$;

Estimate the source-location coordinate $\mathbf{x}_{\mathbf{s}}$;

Calculate background velocity gradient $\nabla_{v} E$;

Update background velocity $v_{0}=v_{0}-\alpha \nabla_{v} E$;

if Objective function stops decreasing;

break;

end if;

end for

on the surface will act as receivers. We use 1, 2, and 4 receiver groups with the true velocity model to get source images. The results are shown in Figure 2b, 2c, and 2d, respectively. The amplitude of all the displayed source images, shown in this paper, is normalized (by dividing the maximum value) between zero and one for the purpose of a better display. We can see that the more receiver groups we use, the better the focusing property of the source image. Using the true velocity to calculate the source image resulted in a focused source image at its true location (the $*$ symbol).

Next, we test how the accuracy of the velocity model influences the source-image quality. We use a simple homogeneous model with a velocity of $2000 \mathrm{~m} / \mathrm{s}$ for this test. The size of the model is $200 \times 200$. The horizontal and vertical grid spacing interval are both $25 \mathrm{~m}$. We use a velocity that is $88 \%$, of the true one, and $120 \%$ of the true velocity to compute the source images. We use one receiver group and autocorrelate the adjoint wavefield to get the source image. This can be expressed as $I[v]=\int_{t} \lambda(t, v) \lambda(t, v) d t$, which is referred 
as the autocorrelation imaging condition (Artman et al., 2010). The source images from the $88 \%$ of true velocity, true velocity, and $120 \%$ of true velocity are shown in Figures $3 \mathrm{a}$, $3 \mathrm{~b}$, and 3c, respectively. We can see that when we use the true velocity to get a source image, the source energy is focused best, and the maximum energy point corresponds to the true source coordinate. When the velocity is lower than the true velocity, the source energy is poorly focused. By comparison, when the velocity is higher than the true velocity, the source energy is better focused than using the lower velocity. This is more apparent in the objective function-value curve in Figure 4. From Figure 4, we can see that this objective function will be minimized when the velocity is accurate. We also notice that this objective function increases fast when the velocity decreases, but it increases slowly when the velocity increases. So this objective function is more sensitive to high velocity perturbations.

\section{Square anomaly}

From the above source-imaging results, we can see that if the velocity is not accurate, the source energy tends to defocus. Thus, we will demonstrate that the proposed method can be used to improve the velocity model and source-image quality, iteratively. First, we analyse the velocity-gradient behavior. We set a homogeneous model of size $200 \times 200$ samples. The background velocity is $2000 \mathrm{~km} / \mathrm{s}$. A source is located in the middle of the model (represented with $*$ symbol), and only one receiver is present on the surface (represented with $\triangle$ symbol). The gradient after considerable Gaussian smoothing is shown in Figure 5 . From the velocity gradient, we can see that the proposed method can achieve smooth velocity updates between source and receiver. Next, we include a square anomaly with a velocity of $3000 \mathrm{~km} / \mathrm{s}$ in the middle, which is shown in Figure 6a. We place a 100 receivers at each of the four boundaries. After 10 iterations of velocity update, the inverted velocity 
is shown in Figure 6b, and a profile at $2500 \mathrm{~m}$ is shown in Figure 7 . The source image using two receiver groups for the background velocity is shown in Figure 8a. Although the acquisition system is perfect, we still fail to get a well-focused source image. We can see that by using the proposed method, the velocity anomaly can be recovered. Using the inverted velocity, the final source image is shown in Figure 8b. From the final source image, we can see that the source energy is perfectly focused on the correct source position with reasonably high resolution. The objective function decreases fast with iterations as shown in Figure 9.

\section{D Marmousi model}

We apply the proposed velocity-update scheme on the Marmousi model. The true velocity model and the acquisition system used in this test are the same as what we used previously in the source-image subsection. We use two receiver groups to calculate the source image. The initial model is a linearly increasing velocity model, shown in Figure 10a. After 40 iterations of velocity update, the inverted velocity model is shown in Figure 10b. The source image corresponding to the initial model is shown in Figure 11a, and the final source image is shown in Figure 11b. We can see that the inverted velocity model has recovered part of the true model with only one single source. Using the improved velocity model, the source energy focuses at its true location. For comparison, we implement the FWI method to invert the velocity and source image simultaneously (Song et al., 2018). We implement the FWI from $2 \mathrm{~Hz}$ to $7 \mathrm{~Hz}$ with a sampling interval of $0.25 \mathrm{~Hz}$ in the frequency domain. The inverted velocity model and final source image are shown in Figures 12a and $12 \mathrm{~b}$, respectively. The starting frequency of $2 \mathrm{~Hz}$ is already very low, which tends to be contaminated with noise in real data. Thus, we still fail to recover a good velocity model, 
even though considerable smoothing is applied to the velocity gradient. The final source image contains more artifacts than the one obtained by the proposed method.

\section{D Overthrust model}

As the proposed method is computationally efficient, it is easy to expand it to 3D models. We further test the proposed method on a modified 3D SEG/EAGE Overthrust model (Aminzadeh et al., 1997) of size $100 \times 100 \times 90$ samples, shown in Figure 13a. The initial velocity model for the inversion test is linearly increasing with depth as shown in Figure 13b. The horizontal and vertical grid-interval spacing is $25 \mathrm{~m}$. We use a Ricker wavelet with a peak frequency of $6 \mathrm{~Hz}$ as a source wavelet. The true source coordinate location is (1000 m, $1250 \mathrm{~m}, 1625 \mathrm{~m})$. We set 400 receivers on the surface distributed like a star shape, which is shown in Figure 14. We use two receiver groups to conduct the inversion process. After 40 iterations, the inverted velocity model is shown in Figure 15. As we can see, the proposed method can recover the main structure of the true model. The source image from the initial velocity model is shown in Figure 16a. From the initial source image, we can see that the source energy cannot focus on the true source location and the vertical resolution is quite low. The inverted velocity model appears more like a smoothed version of the Overthrust model. Using the inverted velocity model, the final source image is shown in Figure 16b. From the final source image, we can see that as the velocity is improved, the source energy focuses on the true source location and the vertical resolution is improved.

\section{D field data}

We now test our proposed method on one event captured in the field. This field data set is collected from an oil field in China during an 11-stage hydraulic fracture treatment. The 
recording system used in this survey consists of 15 levels of three-component geophones. The geometry, which includes a treatment well (blue dots), a monitoring well (red dots), and receivers (black triangles) is shown in Figure 17. It is easy to find one event in the data shown in Figure 18a. As the method we propose is based on the acoustic wave equation, we mute the S-wave and just utilize P-wave which is shown in Figure 18b. We have very limited velocity information from the well log. In order to mitigate the influence from the reflection wave, we use the smoothed the well-log velocity as the initial velocity shown in Figure 19a. After 10 iterations of velocity update with five receiver groups, the inverted velocity is shown in Figure 19b. Using the same number of receiver groups, the initial source image is shown in Figure 20a. We can see that the source energy is poorly focused. Using the inverted velocity, the final source image is shown in Figure 20b. It is obvious that the quality of final source image is much improved, and the source energy is better focused. In order to demonstrate the accuracy of our proposed method, we compare our result with that previously obtained by Tan et al. (2018). These authors used the neighbourhood algorithm to invert for $1 \mathrm{D}$ velocity model and locate the event. Based on their inversion, and the source position is at position $(933.5 \mathrm{~m}, 2710.7 \mathrm{~m})$. This estimated source location is very close to the maximum energy point of the source image we obtained.

\section{DISCUSSIONS}

We use the geometric-mean imaging condition to calculate the source image in our proposed method. The more receiver groups we use, the more focused source image we obtain. If the acquisition system is dense and we still try to use more than four receiver groups, the source image will be very well focused even when the velocity is wrong. Under this condition, what our proposed method can do is limited because there is not much room 
to improve the focusing property of the source image. So choosing the number of receiver groups is important. Our suggestion is that if the acquisition system is dense, we just need to use no more than three receiver groups. Otherwise, if the acquisition system is sparse, more receiver groups are needed to get a good source image initially. The focusing property of the source image behaves differently to the velocity model based on the chosen imaging conditions. When we use the geometric-mean imaging condition, the objective function is less sensitive to the higher velocities. This is one limitation of the proposed method. If another imaging condition is used, we expect that the objective function will behave differently as a function of the velocity.

At each iteration, after we calculate the source image, we need to calculate the estimated source coordinate $\mathbf{x}_{\mathbf{s}}$. For each source image, we can only get one source coordinate which represents the most likely position of one micro-seismic event. This feature leads to another limitation of the proposed method. This method is more suitable for a single point source at a time. If the recorded data contains multiple waves from different micro-seismic events, using the geometric-mean imaging condition, we can locate several events in one source image. Under the condition that these events can be well separated, we can separate the source image which contains multiple events into different source images corresponding to single event, and conduct our method event by event. For the multi-source case, the measurements of multiple sources focusing properties within a single image can be the subject of future research.

\section{CONCLUSIONS}

We introduced a source-focusing function to optimize the velocity model and improve the source image for micro-seismic data. This objective function is used to quantitatively mea- 
sure the focusing property of the source image, and it reaches minimum when the velocity model is accurate. This objective function is immune to the cycle-skipping problem often associated with a bad initial guess, which impedes classic full-waveform implementations. The proposed method is also superior to full-waveform inversion with respect to the computational efficiency, as it does not require inner iterations to update the source image. Using a geometric-mean source-imaging condition, the resulting source-image resolution is high even with sparsely collected data. It also mitigates uncertainties in the source wavelet and origin time. Using a conjugate gradient method to update velocity, the source energy gets more focused and corrects to the true source location. Applications to data generated from the 2D Marmousi and 3D Overthrust models yield reasonably good results. The application on field data also shows that the proposed method can provide more accurate velocity models and improve source-image quality.

\section{ACKNOWLEDGMENTS}

We thank KAUST for its support and the SWAG group for the collaborative environment. We thank Dr.Ye Lin and Dr.Yuyang Tan from University of Science and Technology of China for their fruitful discussions and providing the field data. 


\section{REFERENCES}

Aminzadeh, F., B. Jean, and T. Kunz, 1997, 3D salt and overthrust models: Society of Exploration Geophysicists.

Artman, B., I. Podladtchikov, and B. Witten, 2010, Source location using time-reverse imaging: Geophysical Prospecting, 58, 861-873.

Choi, Y., and T. Alkhalifah, 2011, Source-independent time-domain waveform inversion using convolved wavefields: Application to the encoded multisource waveform inversion: Geophysics, 76, no. 5, R125-R134.

Eisner, L., P. M. Duncan, W. M. Heigl, and W. R. Keller, 2009, Uncertainties in passive seismic monitoring: The Leading Edge, 28, 648-655.

Gajewski, D., and E. Tessmer, 2005, Reverse modelling for seismic event characterization: Geophysical Journal International, 163, 276-284.

Hou, J., and W. Symes, 2018, Inversion velocity analysis in the subsurface-offset domain: Geophysics, no. 2, 83, R189-R200.

Huang, G., and W. Symes, 2016, Matched source waveform inversion: space-time extension: 86th Annual International Meeting, SEG, Expanded Abstracts, 1426-1431.

Kaderli, J., W. D. McChesney, and S. E. Minkoff, 2015, Microseismic event estimation in noisy data via full waveform inversion: 85th Annual International Meeting, SEG, Expanded Abstracts, 1159-1164.

Lin, Y., and H. Zhang, 2016, Imaging hydraulic fractures by microseismic migration for downhole monitoring system: Physics of the Earth and Planetary Interiors, 261, 88-97.

Nakata, N., and G. C. Beroza, 2016, Reverse time migration for microseismic sources using the geometric mean as an imaging condition: Geophysics, 81, no. 2, KS51-KS60.

Plessix, R. E., 2006, A review of the adjoint-state method for computing the gradient of a 
functional with geophysical applications: Geophysical Journal International, 167, 495503.

Rentsch, S., S. Buske, S. Luth, and S. Shapiro, 2004, Location of seismicity using gaussian beam type migration: 74th Annual International Meeting, SEG, Expanded Abstracts, $354-357$.

Sava, P., 2011, Micro-earthquake monitoring with sparsely sampled data: Journal of Petroleum Exploration and Production Technology, 1, 43-49.

Song, C., T. Alkhalifah, and Z. Wu, 2018, Waveform inversion of passive seismic data using multi-scattered energy: 80th EAGE Conference and Exhibition 2018, We B 07.

Song, C., T. Alkhalifah, Z. Wu, and B. Sun, 2017, Non-stationary filter used in microseismic source imaging: 87th Annual International Meeting, SEG, Expanded Abstracts, $2898-2902$.

Song, C., Z. Wu, and T. Alkhalifah, 2019, Passive seismic event estimation using multiscattering waveform inversion: Geophysics, accepted.

Sun, J., Z. Xue, S. Formel, T. Y. Zhu, and N. Nakata, 2016, Full waveform inversion of passive seismic data for sources and velocities: 86th Annual International Meeting, SEG, Expanded Abstracts, 1405-1410.

Tan, Y., C. He, and Z. Mao, 2018, Microseismic velocity model inversion and source location: The use of neighborhood algorithm and master station method: Geophysics, 83, no. 4, KS49-KS63.

Waldhauser, F., and W. Ellsworth, 2000, A double-difference earthquake location algorithm: Method and application to the northern hayward fault, california.: Bulletin of the Seismological Society of America, 1353-1368.

Wang, H. C., and T. Alkhalifah, 2016, Microseismic imaging using a source-independent 
full-waveform inversion method: 86th Annual International Meeting, SEG, Expanded Abstracts, 2596-2600.

Warpinski, N., 2009, Microseismic monitoring: Inside and out: Society of Petroleum Engineers, 61, 80-85.

Witten, B., and J. Shragge, 2017, Image-domain velocity inversion and event location for microseismic monitoring: Geophysics, 82, no. 5, KS71-KS83.

Wu, Z., and T. Alkhalifah, 2017, A new wave equation based source location method with full-waveform inversion: 74th Annual International Conference and Exhibition, EAGE, Extend Abstracts, Th P6 15. 


\section{LIST OF FIGURES}

1 Schematic cartoons of setting receiver groups (triangles with different colors represent different receiver groups, black star represents micro-seismic source).

2 The true Marmousi velocity model; (a) the source image for true velocity using 1 receiver group (b), using 2 receiver groups (c), and using 4 receiver groups (d) (* denotes the true source location).

3 Source images corresponding to when using a velocity that is $88 \%$ of the true velocity (a), the true velocity(b), and $120 \%$ of the true velocity (c) (* denotes the true source location).

4 The objective function as a function of homogeneous velocity models.

5 The velocity gradient for a homogeneous model with a source in the middle and a receiver at the top $(*$ denotes the true source location).

6 Square anomaly (a) and the inverted velocity (b).

7 The velocity profile of true velocity, initial velocity, and inverted velocity at location $2500 \mathrm{~m}$ from Figure 6.

8 The source image from (a) the initial velocity and (b) the inverted velocity with two receiver groups ( $*$ denotes the true source location).

9 The objective-function value as a function of iterations.

10 The initial velocity model (a) and final inverted velocity (b) using two receiver groups for the Marmousi model.

11 The source image from the initial velocity model (a) and final source image from inverted velocity model (b) using two receiver groups for the Marmousi model $(*$ denotes the true source location).

12 The inverted velocity model (a) and final source image from inverted velocity model 
(b) using FWI (* denotes the true source location).

13 The true Overthrust velocity model (a) and initial inverted velocity.

14 The source position and the acquisition system for the Overthrust model $(*$ denotes the true source location and o denotes the receivers position).

15 The inverted velocity using two receiver groups.

16 The source image from the initial velocity model (a) and the final source image from the inverted velocity model (b) using two receiver groups.

17 Field data collecting geometry with treatment well (blue dots), monitoring well (red dots) and receiver (black triangles).

18 Field data example. Recorded field data (a) and data with S-wave muted (b).

19 Smoothed well-log initial velocity with receiver (triangles) geometry (a) and inverted velocity for the field data example(b).

20 Source image from the initial velocity (a) and the inverted velocity (b) using five receiver groups for the field data example. 


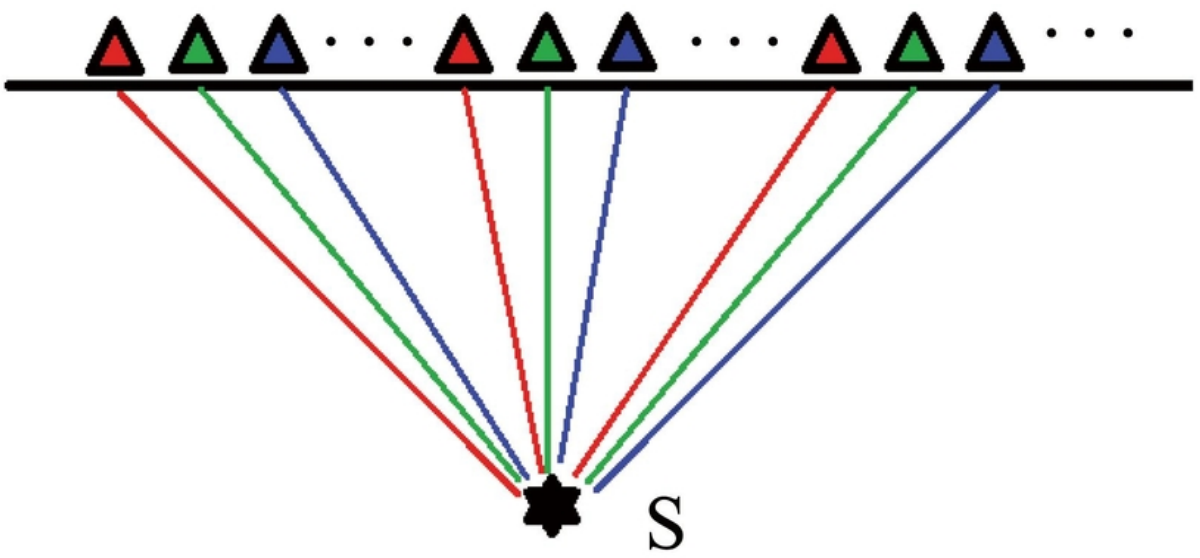

1 Schematic cartoons of setting receiver groups (triangles with different colors represent different receiver groups, black star represents micro-seismic source).

$74 \times 41 \mathrm{~mm}(300 \times 300$ DPI $)$ 

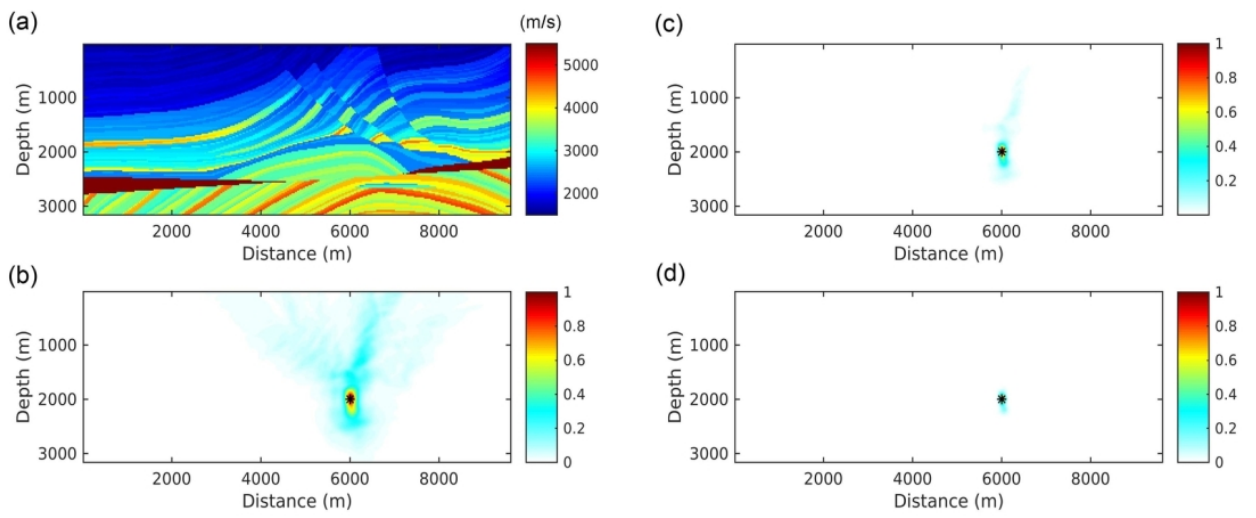

2 The true Marmousi velocity model; (a) the source image for true velocity using 1 receiver group (b), using 2 receiver groups (c), and using 4 receiver groups (d) ( $\$ * \$$ denotes the true source location).

$149 \times 59 m m(300 \times 300$ DPI $)$ 

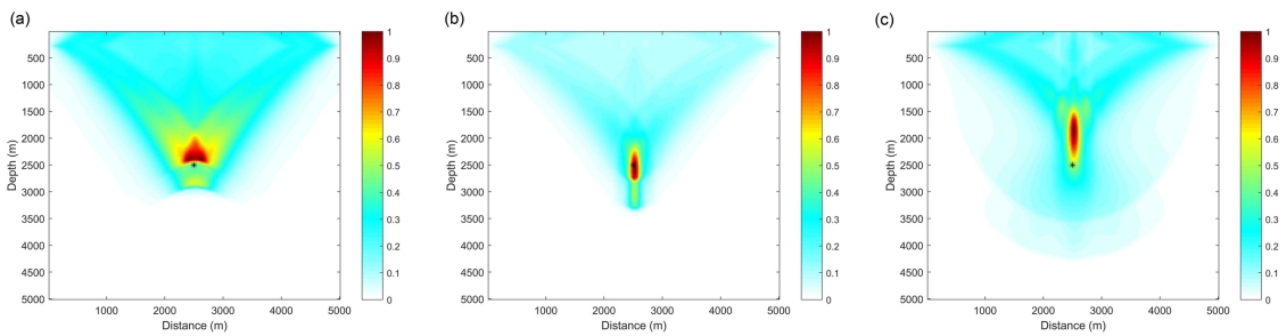

3 Source images corresponding to when using a velocity that is $88 \backslash \%$ of the true velocity (a), the true velocity $(b)$, and $120 \backslash \%$ of the true velocity (c) ( $\$ * \$$ denotes the true source location).

$222 \times 56 \mathrm{~mm}(300 \times 300 \mathrm{DPI})$

This paper presented here as accepted for publication in Geophysics prior to copyediting and composition.

(C) 2019 Society of Exploration Geophysicists. 


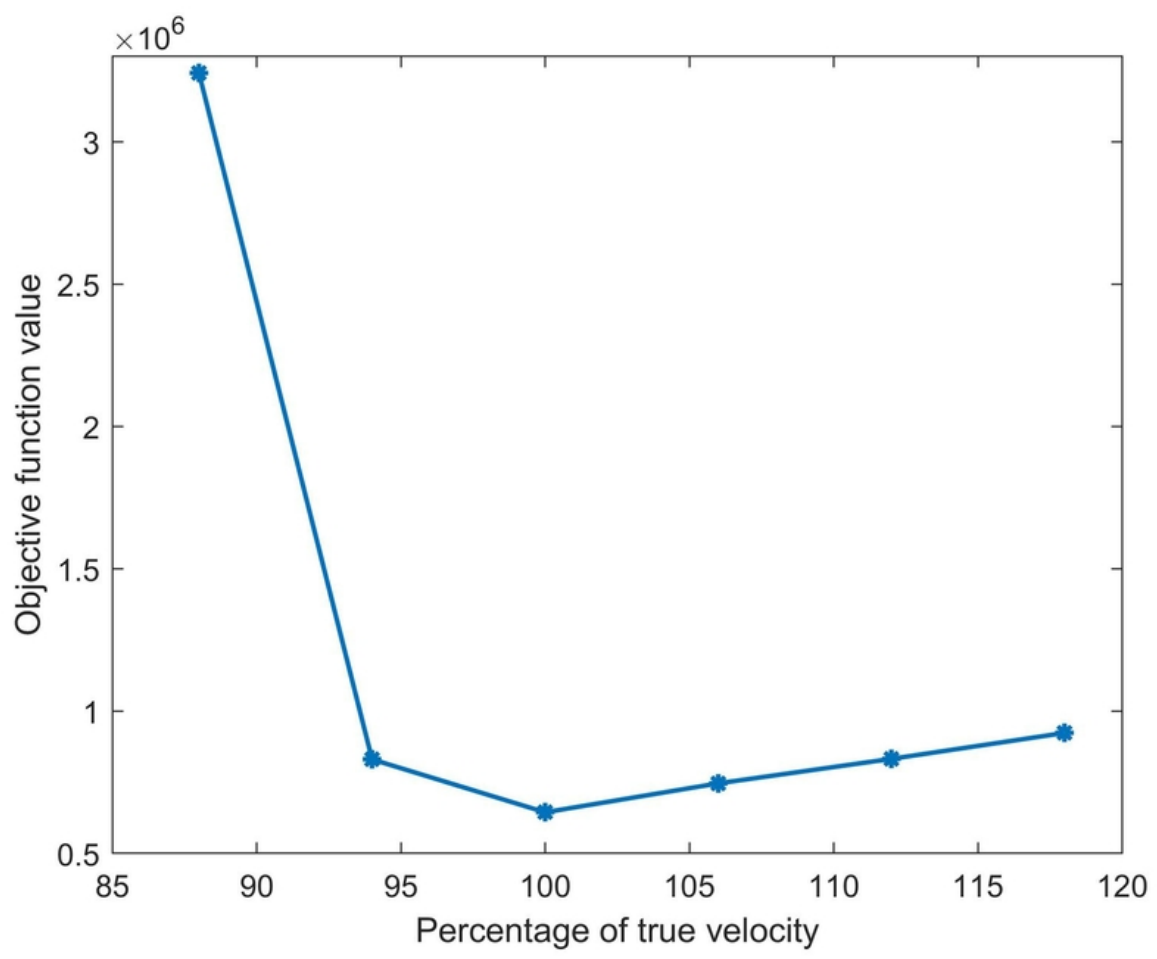

4 The objective function as a function of homogeneous velocity models.

$74 \times 56 \mathrm{~mm}$ ( $300 \times 300$ DPI)

This paper presented here as accepted for publication in Geophysics prior to copyediting and composition.

(C) 2019 Society of Exploration Geophysicists. 


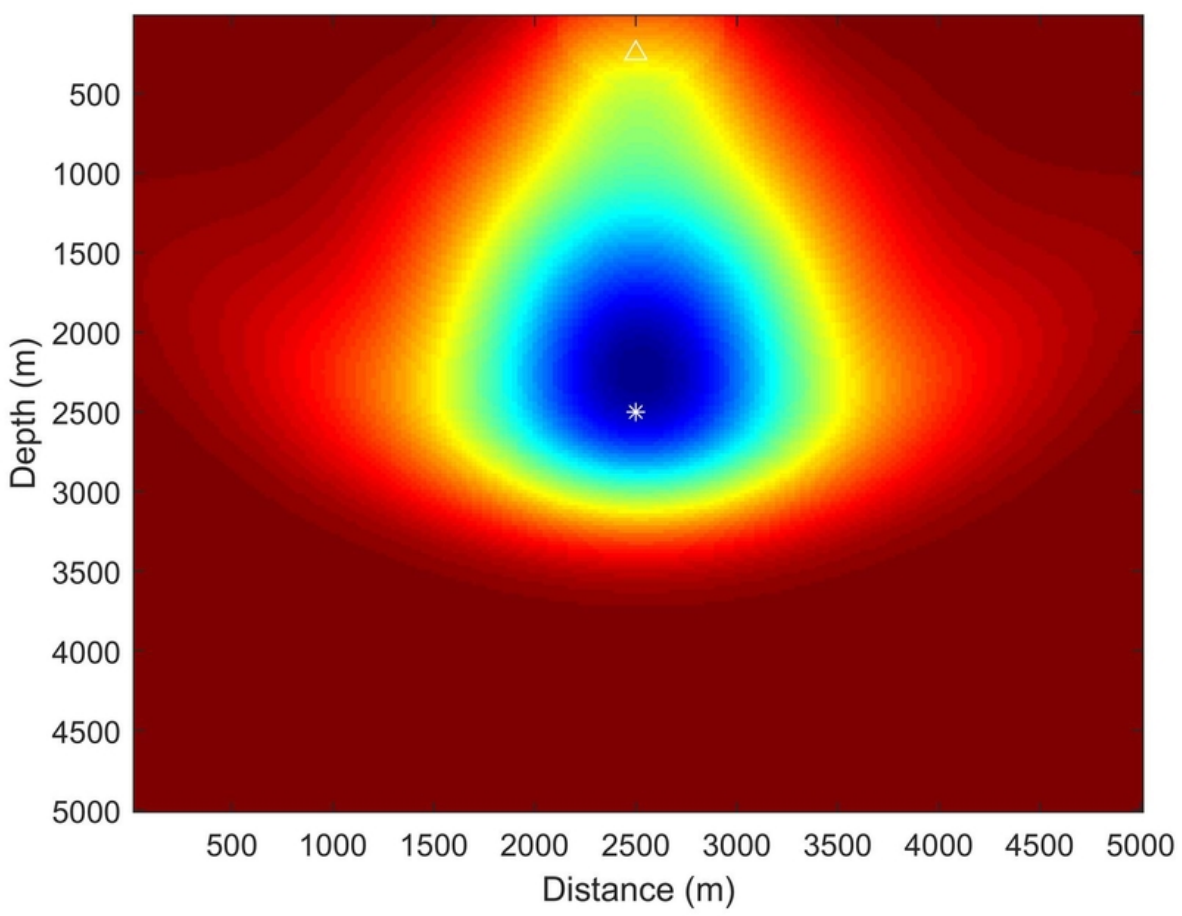

5 The velocity gradient for a homogeneous model with a source in the middle and a receiver at the top ( $\$ * \$$ denotes the true source location).

$74 \times 56 \mathrm{~mm}(300 \times 300 \mathrm{DPI})$ 

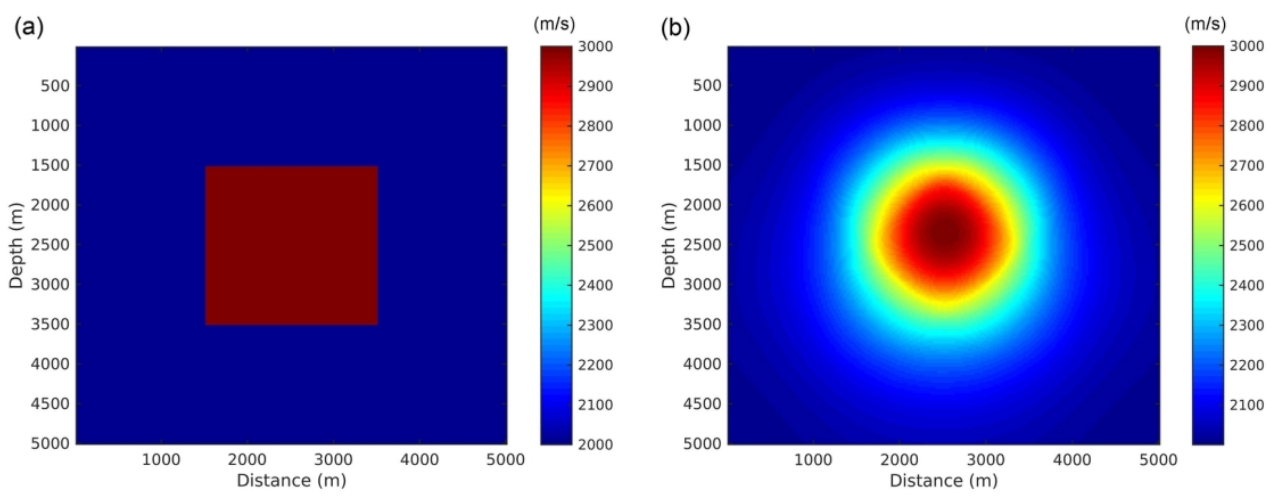

6 Square anomaly (a) and the inverted velocity (b).

$149 \times 56 \mathrm{~mm}(300 \times 300 \mathrm{DPI})$ 


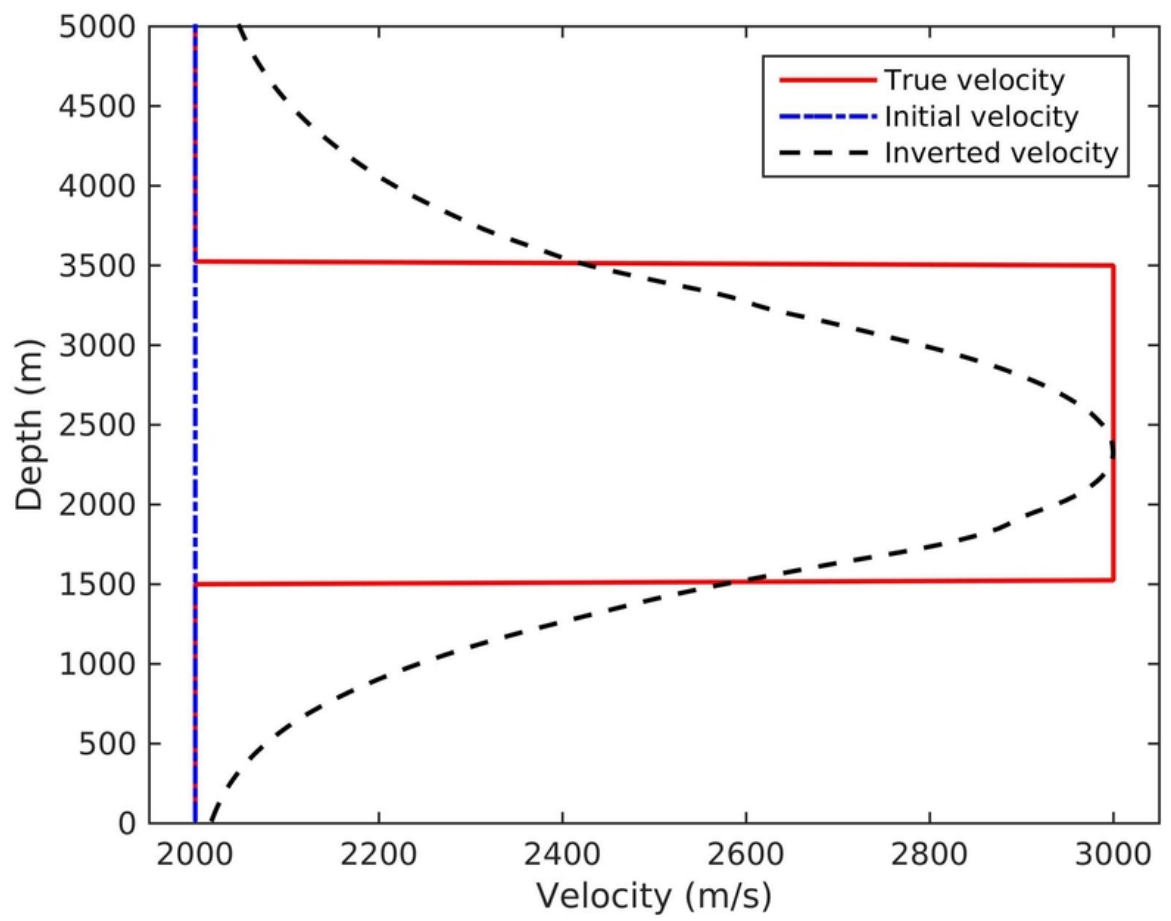

7 The velocity profile of true velocity, initial velocity, and inverted velocity at location $2500 \mathrm{~m}$ from Figure $\sim \backslash$ ref $\{$ fig:Figure6.eps $\}$.

$74 \times 56 \mathrm{~mm}(300 \times 300 \mathrm{DPI})$

This paper presented here as accepted for publication in Geophysics prior to copyediting and composition. (C) 2019 Society of Exploration Geophysicists. 
(a)

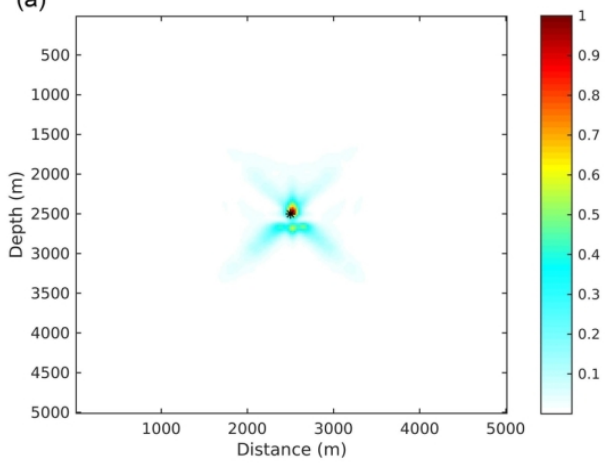

(b)

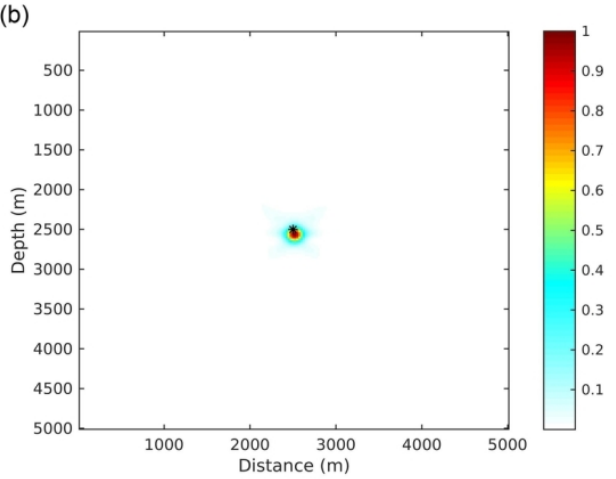

8 The source image from (a) the initial velocity and (b) the inverted velocity with two receiver groups ( $\$ * \$$ denotes the true source location).

$149 \times 56 \mathrm{~mm}(300 \times 300 \mathrm{DPI})$

This paper presented here as accepted for publication in Geophysics prior to copyediting and composition.

(C) 2019 Society of Exploration Geophysicists. 


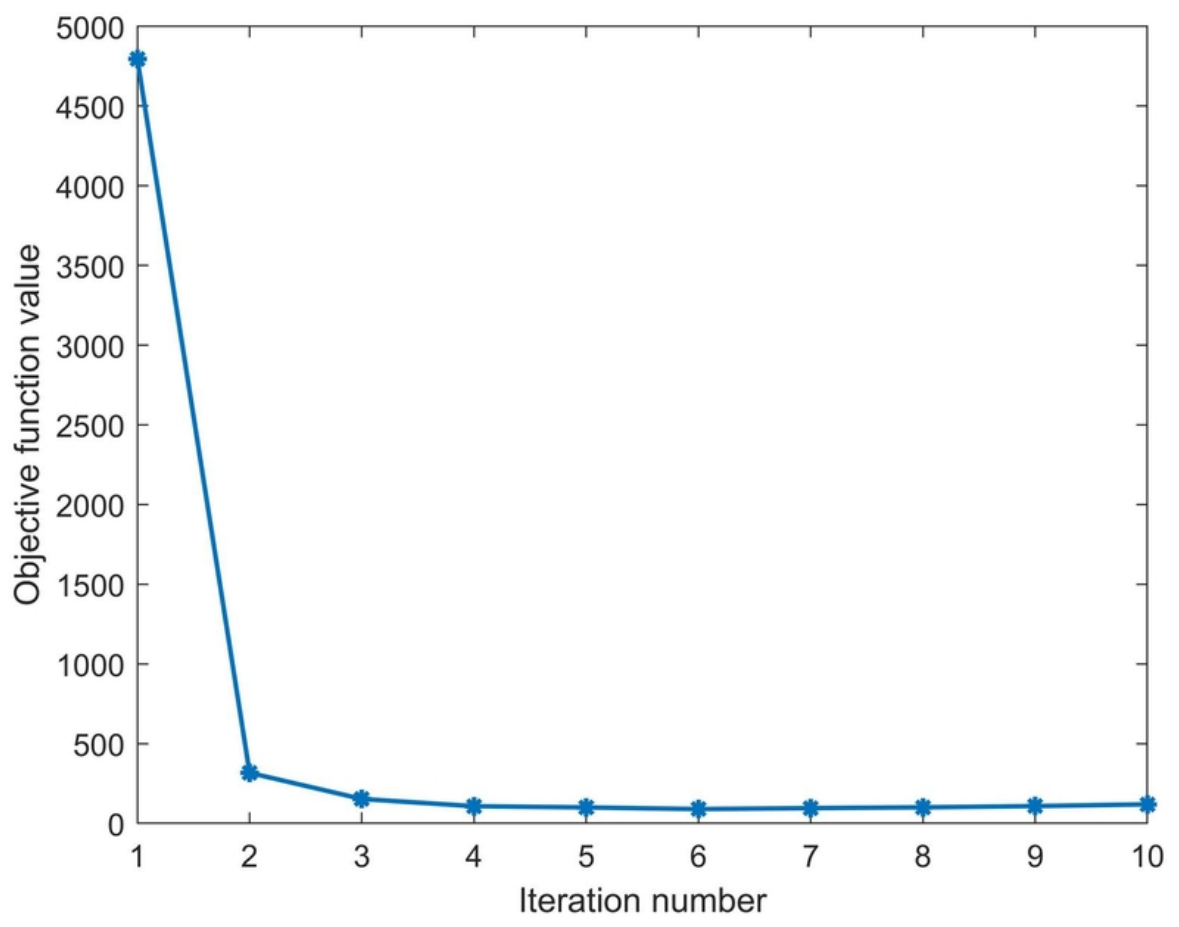

9 The objective-function value as a function of iterations.

$74 \times 56 \mathrm{~mm}(300 \times 300 \mathrm{DPI})$

This paper presented here as accepted for publication in Geophysics prior to copyediting and composition. (C) 2019 Society of Exploration Geophysicists. 
(a) $(\mathrm{m} / \mathrm{s})$

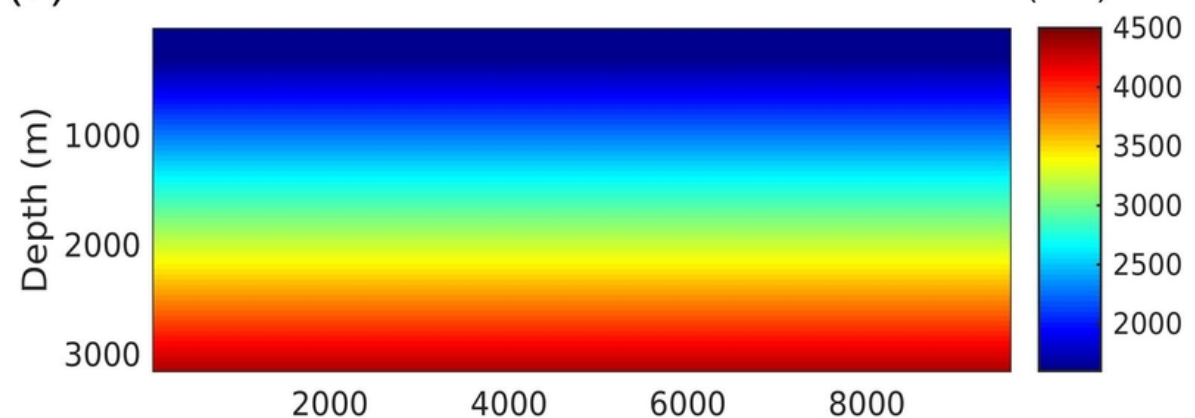

(b)

Distance $(m)$

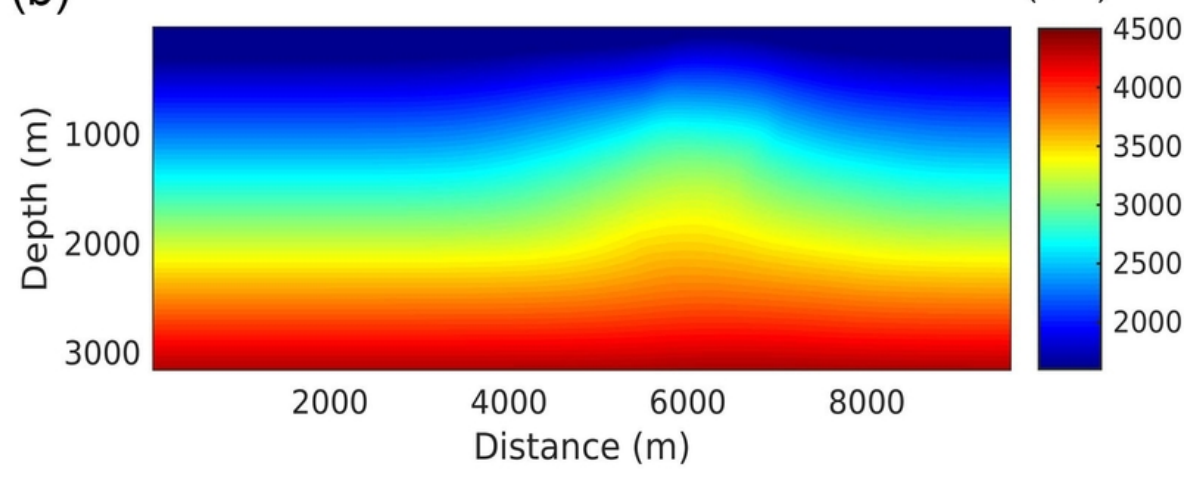

10 The initial velocity model (a) and final inverted velocity (b) using two receiver groups for the Marmousi model.

$74 \times 59 \mathrm{~mm}(300 \times 300 \mathrm{DPI})$ 
(a)

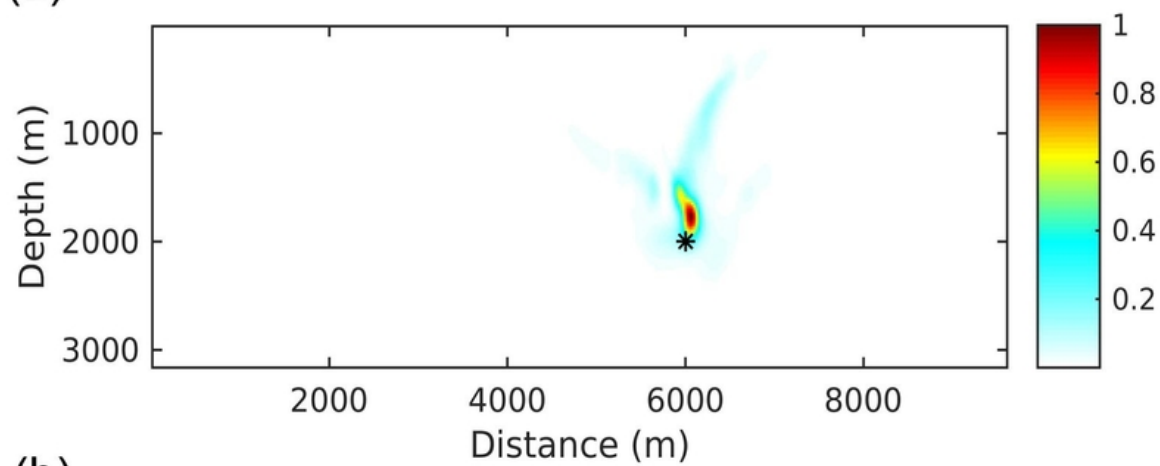

(b)

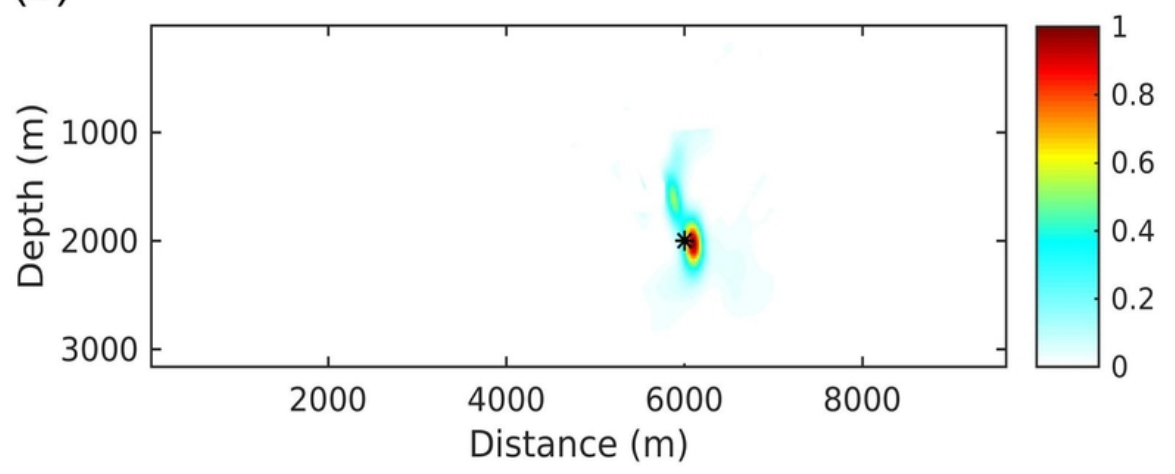

11 The source image from the initial velocity model (a) and final source image from inverted velocity model (b) using two receiver groups for the Marmousi model ( $\$ * \$$ denotes the true source location).

\section{$74 \times 59 \mathrm{~mm}(300 \times 300$ DPI $)$}




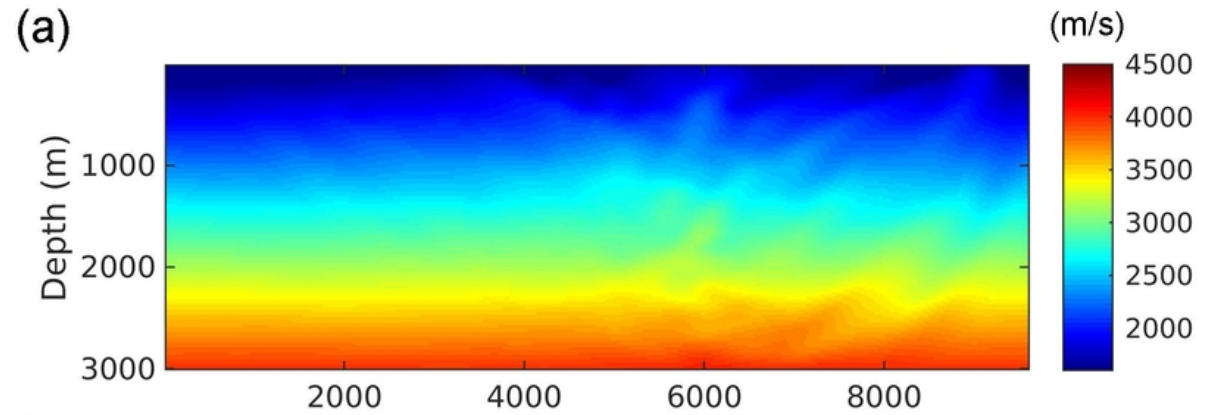

(b)

Distance $(\mathrm{m})$

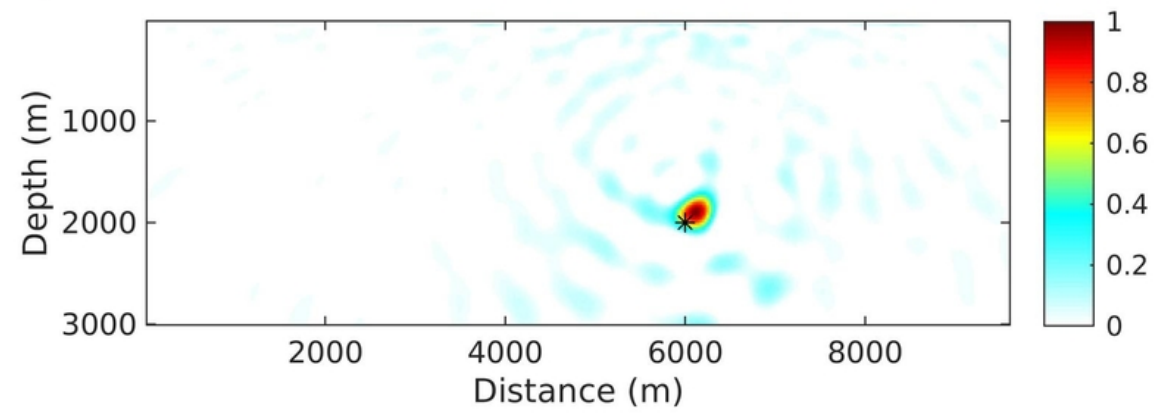

12 The inverted velocity model (a) and final source image from inverted velocity model (b) using FWI ( $\$ * \$$ denotes the true source location).

$75 \times 51 \mathrm{~mm}(300 \times 300 \mathrm{DPI})$ 

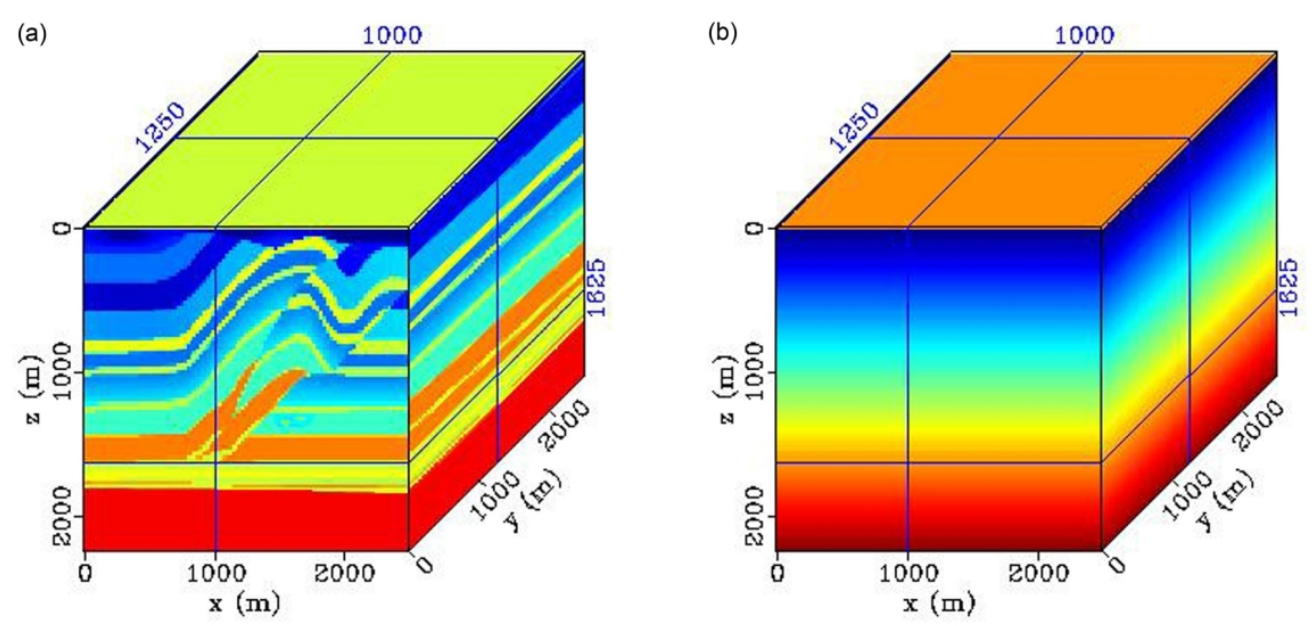

13 The true Overthrust velocity model (a) and initial inverted velocity.

$$
149 \times 71 \mathrm{~mm}(300 \times 300 \text { DPI })
$$




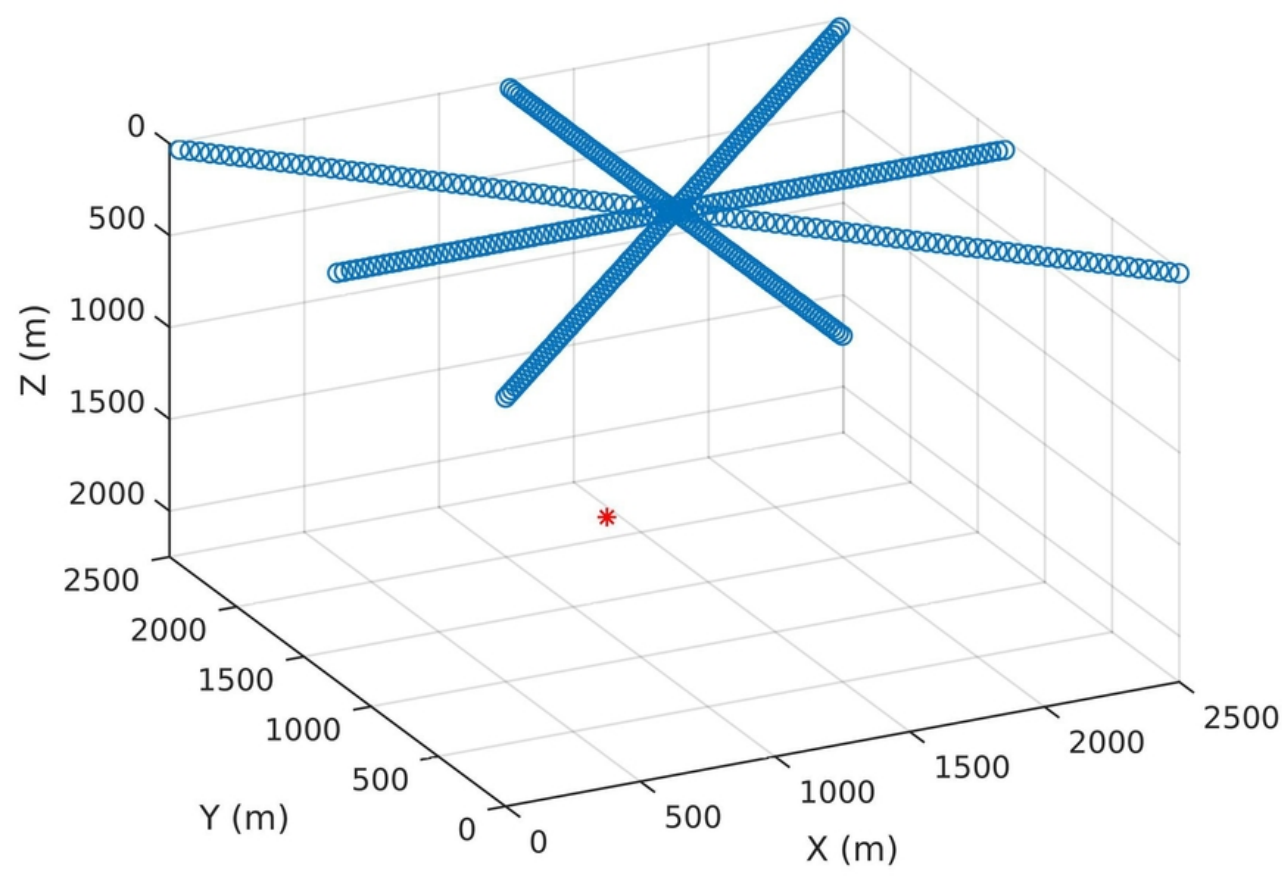

14 The source position and the acquisition system for the Overthrust model ( $\$ * \$$ denotes the true source location and $\$ \backslash$ circ $\$$ denotes the receivers position).

$74 \times 55 \mathrm{~mm}(300 \times 300 \mathrm{DPI})$

This paper presented here as accepted for publication in Geophysics prior to copyediting and composition. (C) 2019 Society of Exploration Geophysicists. 


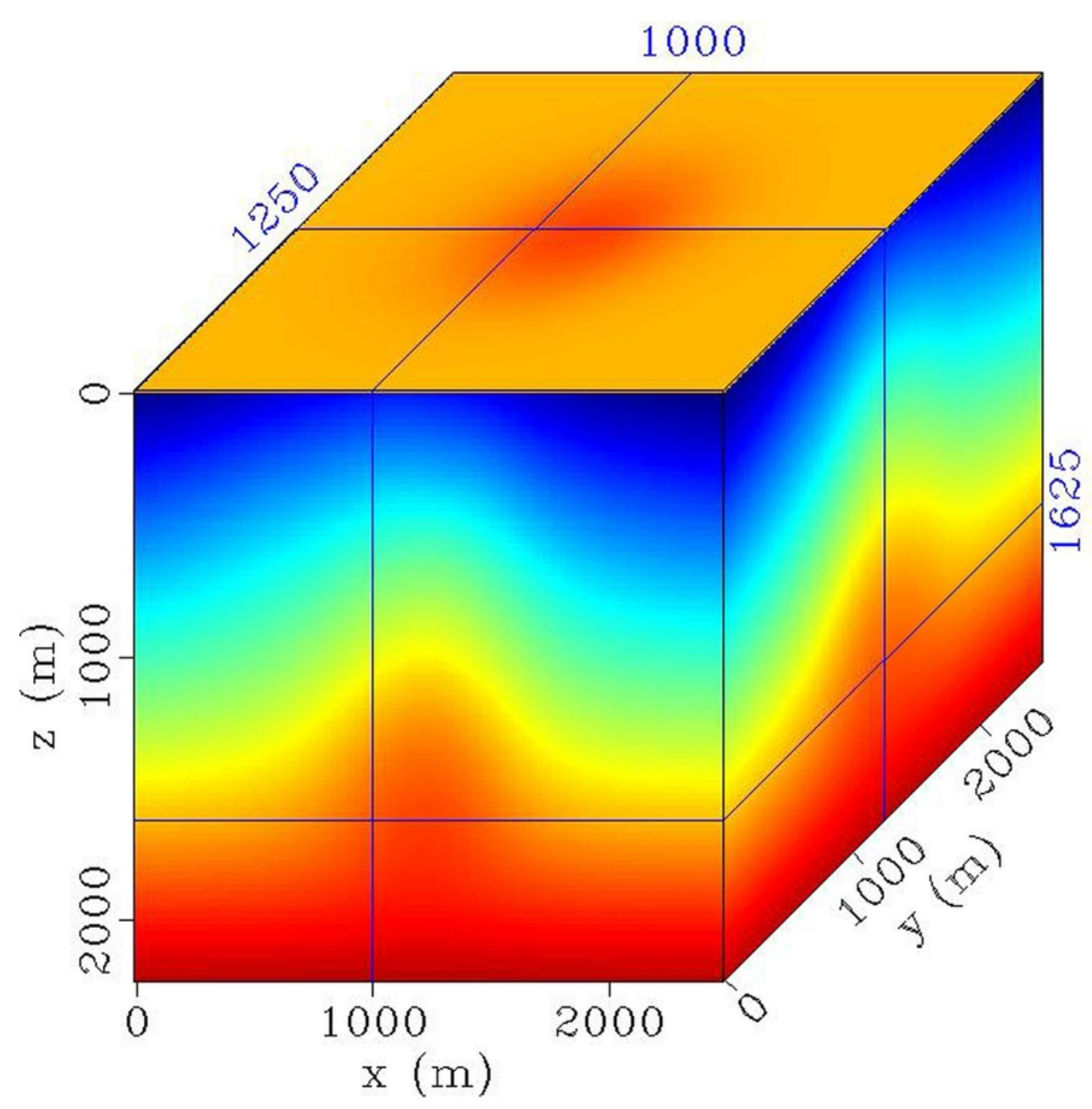

15 The inverted velocity using two receiver groups.

$74 \times 73 \mathrm{~mm}(300 \times 300 \mathrm{DPI})$

This paper presented here as accepted for publication in Geophysics prior to copyediting and composition. (C) 2019 Society of Exploration Geophysicists. 
(a)

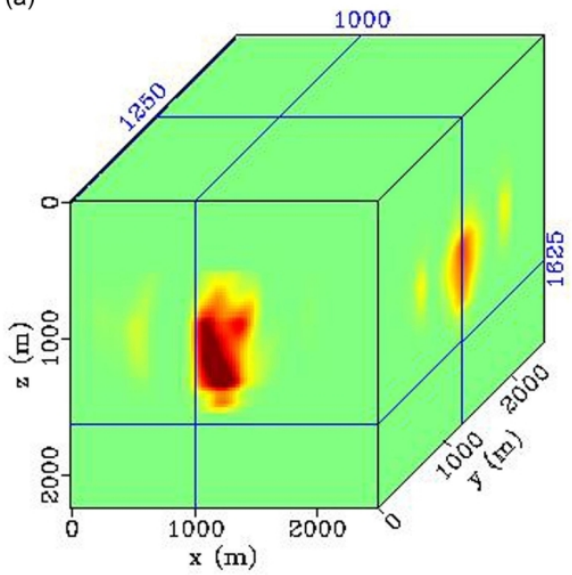

(b)

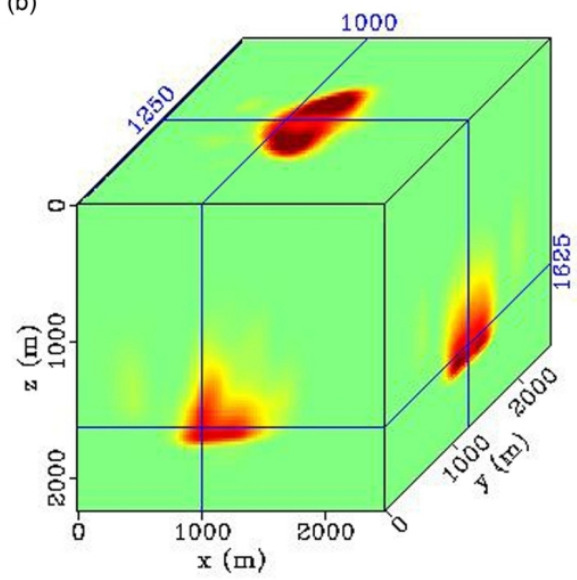

16 The source image from the initial velocity model (a) and the final source image from the inverted velocity model (b) using two receiver groups.

$149 \times 69 \mathrm{~mm}(300 \times 300$ DPI $)$ 


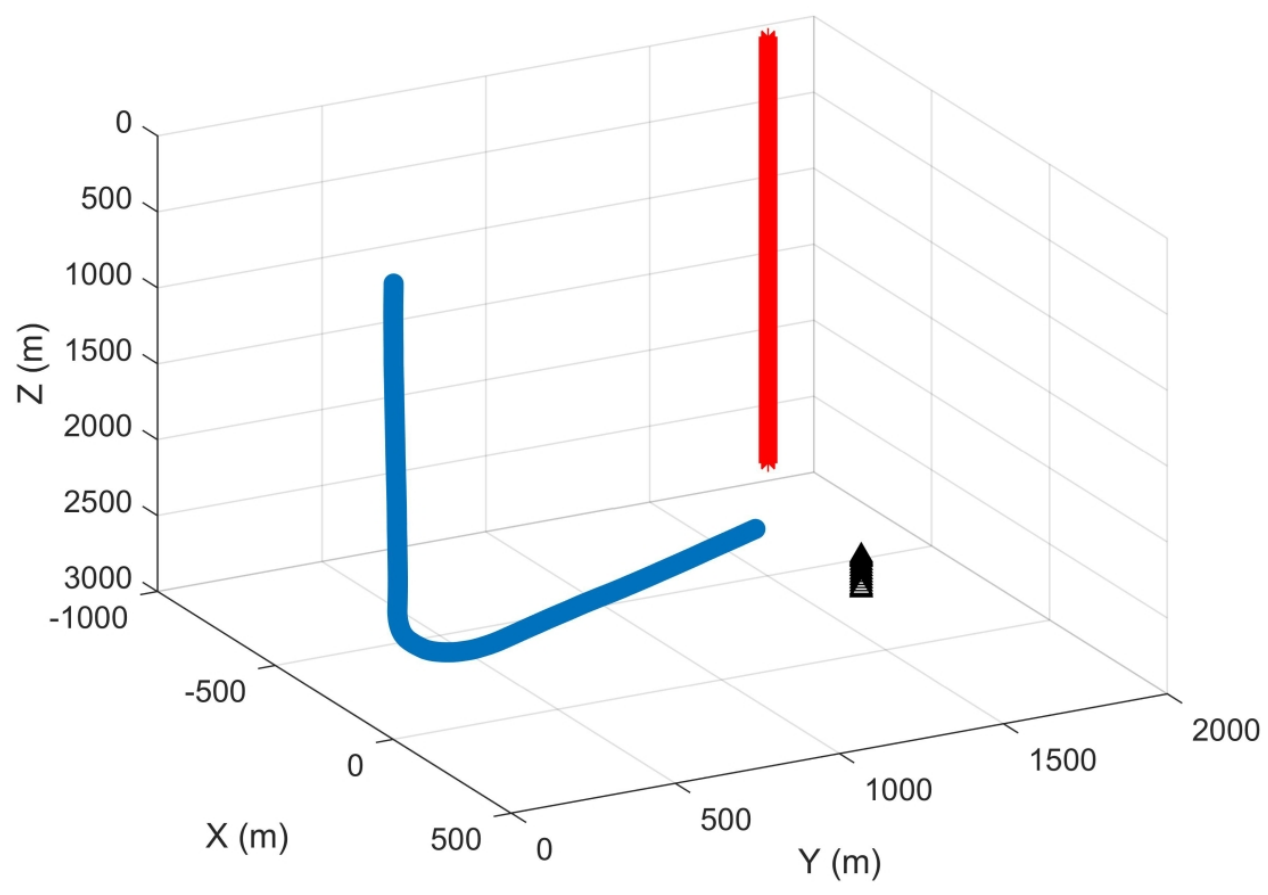

17 Field data collecting geometry with treatment well (blue dots), monitoring well (red dots) and receiver (black triangles).

$1234 \times 926 \mathrm{~mm}(600 \times 600 \mathrm{DPI})$

This paper presented here as accepted for publication in Geophysics prior to copyediting and composition.

(C) 2019 Society of Exploration Geophysicists. 

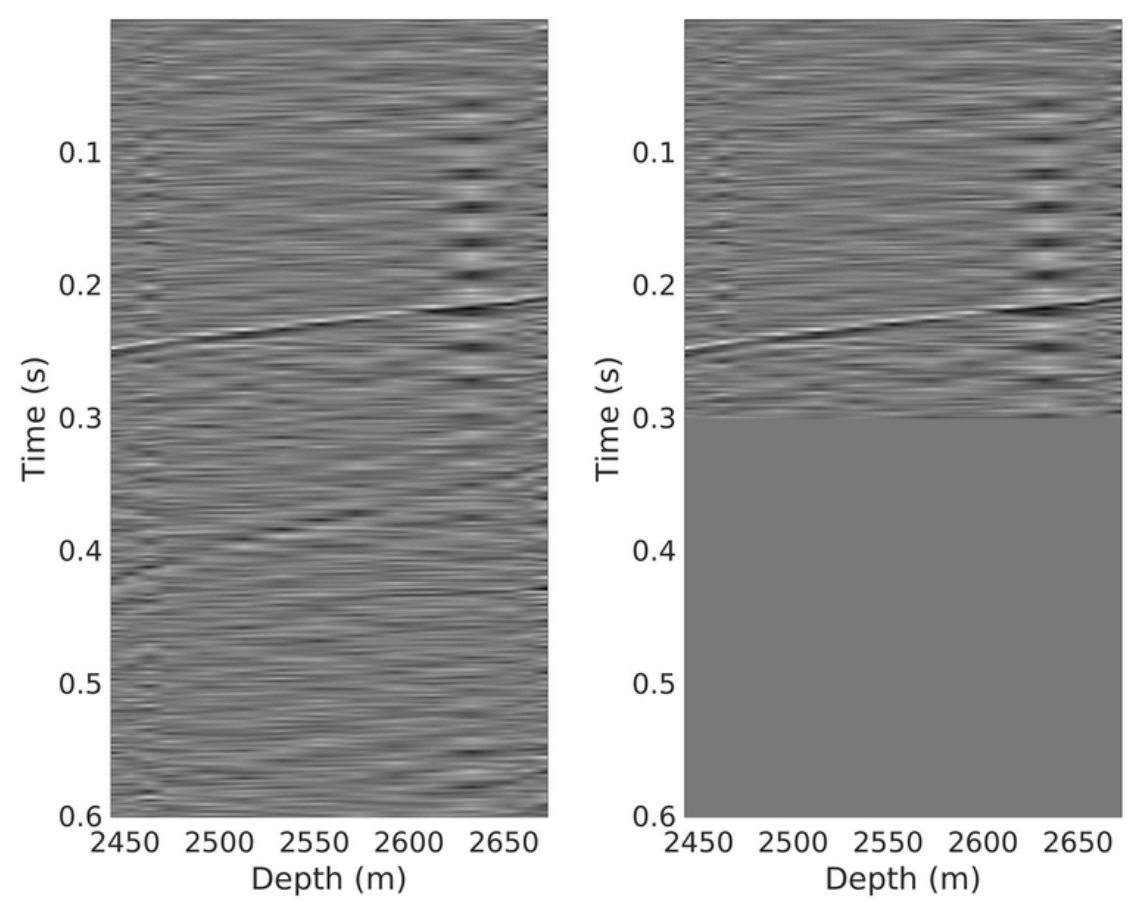

18 Field data example. Recorded field data (a) and data with S-wave muted (b).

$74 \times 56 \mathrm{~mm}(300 \times 300 \mathrm{DPI})$

This paper presented here as accepted for publication in Geophysics prior to copyediting and composition.

(C) 2019 Society of Exploration Geophysicists. 

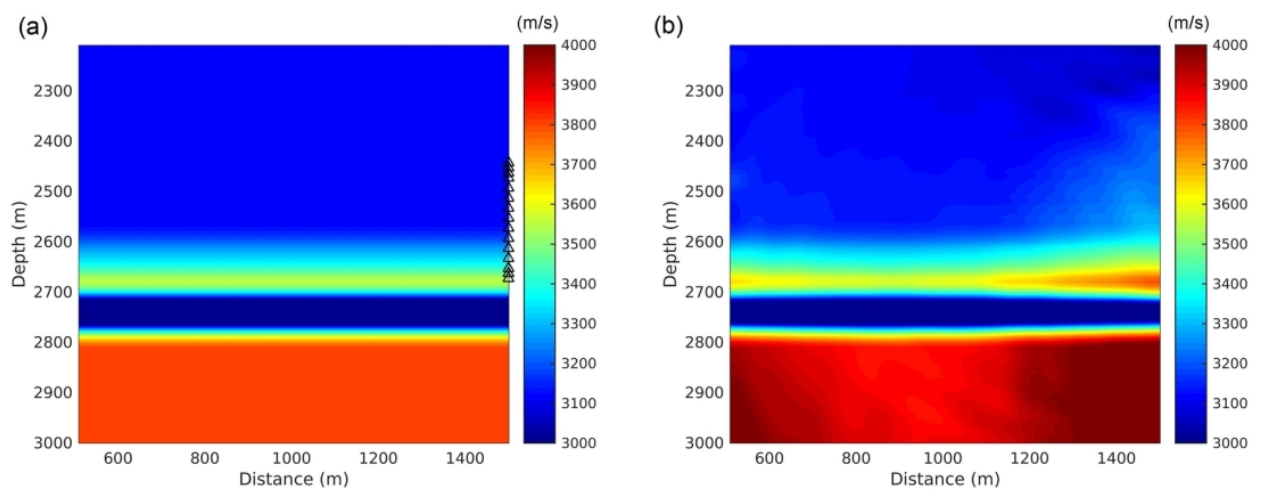

19 Smoothed well-log initial velocity with receiver (triangles) geometry (a) and inverted velocity for the field data example(b).

$149 \times 56 \mathrm{~mm}(300 \times 300 \mathrm{DPI})$ 
(a)

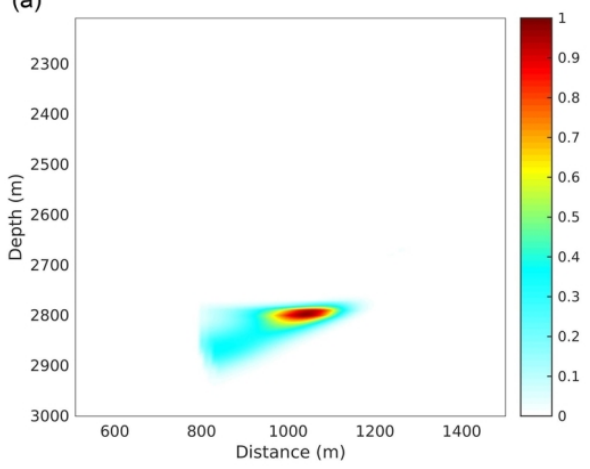

(b)

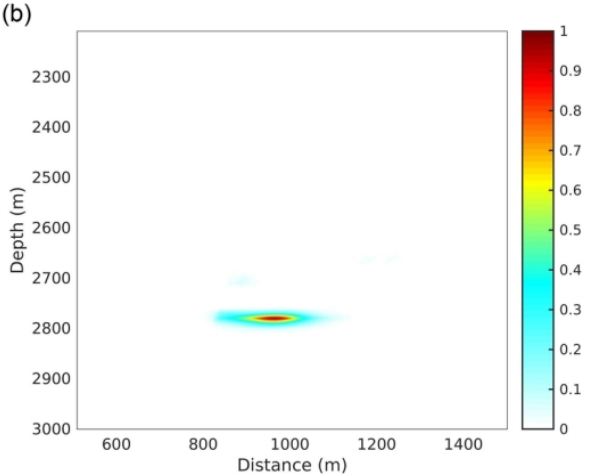

20 Source image from the initial velocity (a) and the inverted velocity (b) using five receiver groups for the field data example.

$149 \times 56 \mathrm{~mm}(300 \times 300$ DPI $)$

This paper presented here as accepted for publication in Geophysics prior to copyediting and composition.

(C) 2019 Society of Exploration Geophysicists. 
DATA AND MATERIALS AVAILABILITY

Data associated with this research are confidential and cannot be released. 